\title{
Matrix-free Laser Desorption/Ionization on Vertically Aligned Carbon Nanotube Arrays
}

\author{
Su Jin Shin, Doo-Won Choi, Ho-Seok Kwak, Goo Il Lim, and Young S. Choi \\ Department of Chemistry, Inha University, Incheon 402-751, Korea. "E-mail: yschoi@imha.ac.kr \\ Received December 17, 2005
}

Key Words : Laser desorption/ionization, Carbon nanotube arrays

The matrix-assisted laser desorption/ionization (MALDI) has established its position as one of the most useful ionization techniques in mass spectrometry ${ }^{1}$ and its contribution to the scientific communities related to mass spectrometry was appreciated with the 2002 Nobel Prize in Chemistry given to the first developer, Tanaka. ${ }^{2}$ It is really an effective and sensitive ion formation technique which commonly employs an organic acid as the matrix. The use of such organic acids as the matrices has provided the high efficiency of desorption and ionization of large molecules including peptides, proteins, synthetic polymers, etc. for the MALDI technique. Interestingly, the matrix, which made the MALDI technique practical, poses an intrinsic constraint to the analyses of relatively small molecules below $1000 \mathrm{Da}$ because the low mass regions in MALDI mass spectra are dominated by the strong background signals of the matrix molecule, its dimer, and their clusters with solvent molecules. Several approaches have been tried to find the methods that give the high desorption/ionization efficiency of MALDI without using the organic matrices. ${ }^{3-7}$

Probably the most successful matrix-free laser desorption/ jonization technique must be the use of porous silicon as a replacement of the matrix. ${ }^{3}$ The desorption/ionization on porous silicon(abbreviated as DIOS) was found to be highly sensitive for the sample below $3,000 \mathrm{Da}$ and showed no interference at all in this low mass region, and is now commercially available. Although the DIOS has been very successful, it has a non-negligible shortcoming that its performance deteriorates with time after fabrication. The degradation may be slowed down by derivatization of the surface but cannot be completely eliminated. ${ }^{4}$

In this Note, we report that the vertically grown carbon nanotube arrays may be used as a substrate for matrix-free laser desorption/ionization of relatively small molecules. It turned out to be highly sensitive for small peptides near and below 2,000 Da and its ionization efficiency does not change after a long-time storage in the room atmosphere.

\section{Experimental Section}

The Vertically Aligned Carbon Nanotubes Arrays. The carbon nanotube arrays (abbreviated as CNTa) grown on a stainless steel plate was purchased from NanoLab (Newton, MA, USA). The substrate size was $10 \mathrm{~mm} \times 10 \mathrm{~mm}$ and thickness $0.25 \mathrm{~mm}$. The $\mathrm{CNTa}$ were fabricated by the
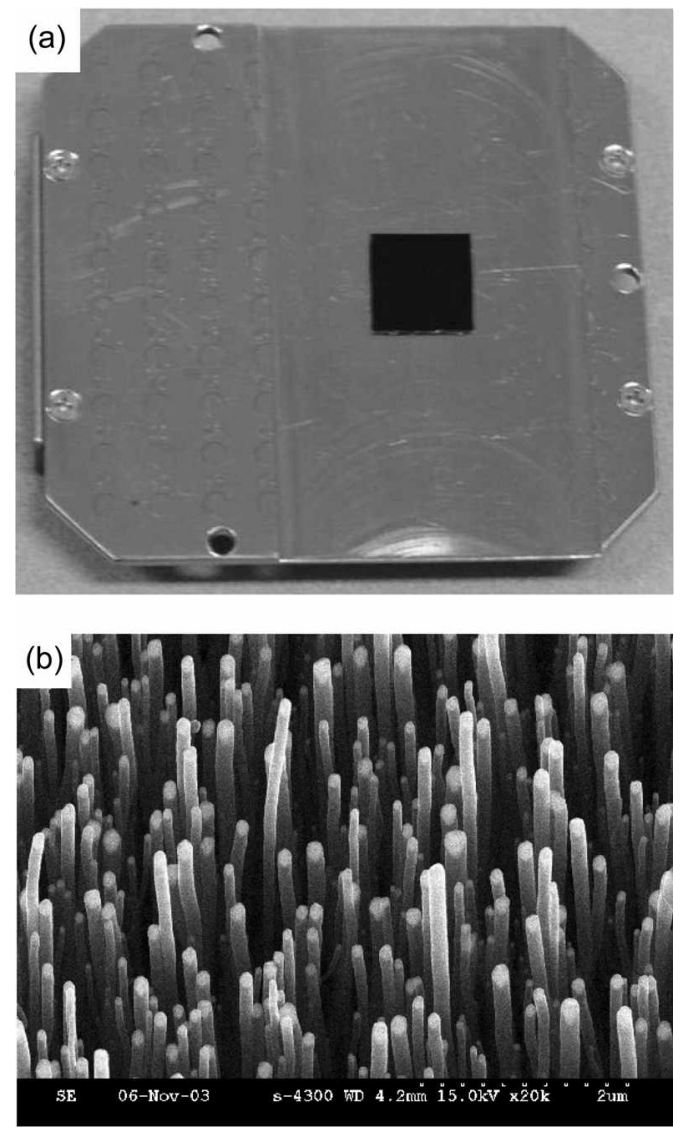

Figure 1. The vertically aligned carbon nanotube arrays (CNTa) used in this work. (a) the substrate mounted on a modified 100-well MALDI sample plate and (b) the SEM image $(\times 20,000$-fold $)$ of the CNTa.

plasma enhanced chemical vapor deposition (PECVD) method using $\mathrm{Cr}$ as a catalyst. The $\mathrm{CNTa}$ were characterized with a scanning electron microscopy (SEM, Hitachi S-4300) to be $4 \mu \mathrm{m}$ long and about $100 \mathrm{~nm}$ in diameter as shown in Figure 1. The number density of the nanotubes was $\sim 5 \times 10^{6} /$ $\mathrm{cm}^{2}$. The substrate plate was attached to a modified 100-we]l MALDI sample plate by using an adhesive tape (Figure 1).

Materials and Sample Preparation. Substance P, DesArg-Bradykinin, and sodjum chloride were purchased from Sigma (St.Louis, MO, USA). The HPLC grade water was obtained from Fisher Scientific Korea Ltd. (Seoul, Korea) and used without further purification. Substance $P$ was dissolved in water at a concentration of $3 \times 10^{-5} \mathrm{M}$ and the 
sodium chloride solution was prepared at a concentration of $0.1 \mathrm{M}$. The analyte solution was mixed with the sodium chloride solution in a volume ratio of $9: 1$. Des-ArgBradykinin dissolved in water at concentration of $\mathrm{I} \times 10^{-9}$ $\mathrm{M}$. The $1 \mu \mathrm{L}$ aliquots of the analytes were dropped onto the $\mathrm{CNTa}$ and dried in air before laser desorption/ionization TOF-MS analysis.

MALDI-TOF-MS Instrument. The mass spectrometric experiments were performed with a time-of-flight mass spectrometer (Voyager DE-STR, Applied Biosystems, Framingham, MA, USA) in the positive ion mode. Desorption/ionization was performed with a $337 \mathrm{~nm}$ nitrogen laser with a $3 \mathrm{~ns}$ pulse width. Available accelerating potential was $+25 \mathrm{kV}$. Laser power was adjusted to give the reasonably good resolution and signal-to-noise (S/N) ratios and each spectrum was accumulated for 100 laser shots.

\section{Results and Discussion}

Matrix-free laser desorption/ionization (LDI) time-of-flight mass spectra of small peptides on the CNTa surface were successfully obtained. Figures 2 and 3 show the matrix-free LDI time-of-flight mass spectra of two small peptides, DesArg-Bradykinin (MW $=904.0 \mathrm{Da})$ and Substance $\mathrm{P}(\mathrm{MW}=$ $1347.6 \mathrm{Da}$ ), respectively. The amounts of the sample peptides dropped on the vertically aligned CNTa were 10 pmol for Des-Arg-Bradykinin and 30 pmol for Substance P, respectively. The detection limit for Substance $P$ was measured to be $\sim 10 \mathrm{fmol}$.

In 2003, Xu et al. reported that they had obtained the matrix-free LDI time-of-flight mass spectra of some small organic molecules and peptides dropped on the stack of carbon nanotubes. ${ }^{7}$ Their substrate, however, was quite different from ours; theirs was a pile of long, tangled nanotubes like a spaghetti but our CNTa consists with the well-aligned, vertically grown nanotubes. More importantly, the ion generation efficiency of the vertically aligned CNTa substrate is much higher than their spaghetti-type one

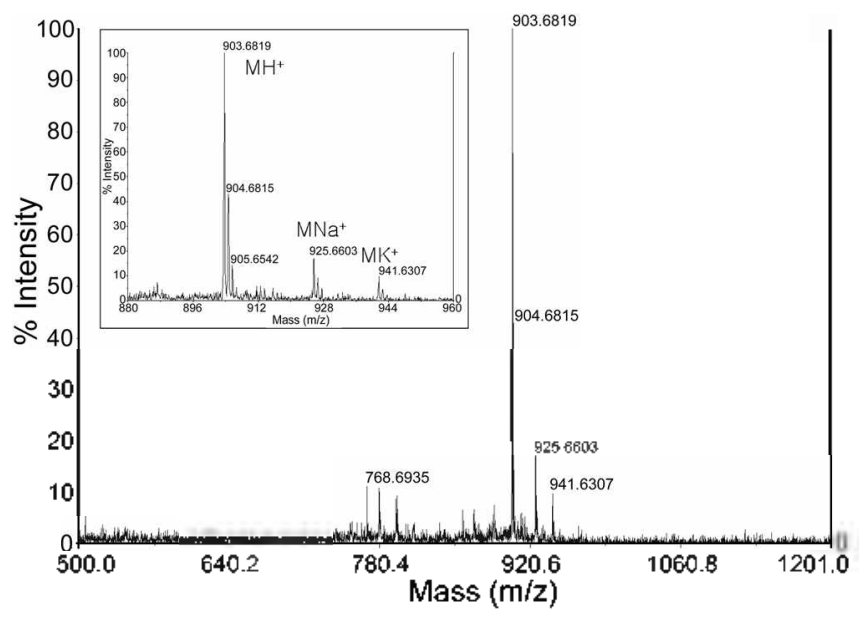

Figure 2. Matrix-free LDI TOF mass spectrum of Des-ArgBradykinin (MW $=904.0 \mathrm{Da}$ ) dropped on the CNTa. The inset is the enlargement of the main ion peaks. although a quantitative comparison is not possible because our analytes were different from theirs. Hence, our results demonstrate that the vertically aligned nanotubes are much more efficient in matrix-free LDI of small peptides than the tangled ones. The DIOS shows much higher ion generation efficiency than our method. ${ }^{3+}$ Considering the fact that our method is not fully optimized, however, the LDI from small peptides dropped on the vertically aligned CNTa looks quite promising.

In order to examine the stability of the vertically aligned CNTa surface, we took the spectra of the same peptide samples after keeping the CNTa substrate in the room atmosphere for a month, and the spectra did not show any noticeable difference from those taken a month earlier. This observation is in sharp contrast to the DIOS which shows a serious degradation with time. ${ }^{4}$ The stability of the CNTa substrate for matrix-free LDI was anticipated from the early observed stability of the carbon nanotubes in air. ${ }^{8}$ This stability of the CNTa substrates suggests that those may be a practically applicable substrate for matrix-free LDI of small organic and peptide molecules. In addition, the vertically aligned CNTa can be grown and patterned routinely with the well established semiconductor processing technologies.?

The mass spectra were obtained for a larger peptide, Insulin $(\mathrm{MW}=5,733 \mathrm{Da})$ at a much lower sensitivity than the smaller peptides. The same observation was reported for $\mathrm{DIOS}^{3+}$ and the unaligned, spaghetti-type nanotube substrates, ${ }^{7}$ and thus it may be a common characteristics of the matrix-free LDI method.

At this point it is necessary to discuss the mechanism of the LDI of peptides from the vertically aligned CNTa substrate. Even though it seems implausible to confirm a reasonable mechanism based on just our results, we suggest that the local heating of the tips of nanotubes by absorption of the laser light may be the primary step. Since the nanotubes strongly absorb the $337 \mathrm{~nm}$ ultraviolet light, ${ }^{10}$ the tips of nanotubes exposed to the laser light would absorb the UV photons. The photon energy, thus deposited on the tips

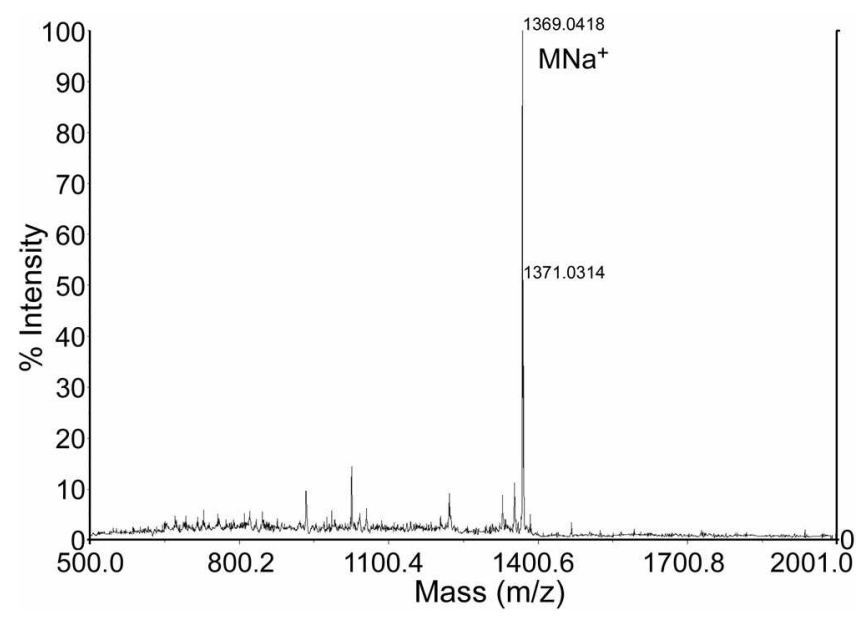

Figure 3. Matrix-free LDI TOF mass spectrum of Substance P (MW $=1,347.6 \mathrm{Da})$ dropped on the CNTa. The dominant ions are the sodium ion adducts. 
of nanotubes, will heat up the tips to a high temperature and the adsorbed molecules on the tips of nanotubes will thermally desorb from the surfaces of the nanotubes. Since the tube is fairly long with respect to the diameter of the nanotubes and each nanotube is well separated from others, the relaxation of the initially reached high temperature will be slow, which enhances the themnal desorption/ionization efficiency. This mechanism reasonably explains why the vertically aligned nanotubes used in this work are more efficient in desorption/ionization than the spaghetti-type stacks of long nanotubes. In the stack of long nanotubes, the initially localized heat to a specific region of a nanotube would flow more rapidly to other parts through the intra- and the inter-tube energy transfer than the vertically aligned CNTa.

Several carbon nanotube arrays that are different in lengths, diameter, number density, method of growth were tested. We found that the closely packed tubes longer than $100 \mu \mathrm{m}$ gave almost no mass signal and the diameter and the method of growth of the tubes does not significantly affect the desorption/ionization efficiency. Probably the close packing and long length of the nanotubes seem to enhance the cooling of the heated tips, supporting our mechanism of local heating. Of the various nanotube substrates, the vertically aligned CNTa shown in Figure 1 demonstrated the best performance of all in terms of sensitivity and stability.

It is interesting to note that the sodium ion adduct is the dominant ionic species for Substance $\mathrm{P}$ as shown in Figure 3. Even for Des-Arg-Bradykinin, the $\mathrm{Na}^{+}$and $\mathrm{K}^{+}$adducts are relatively strong compared to the mass spectra obtained with the matrices of organic acids.(the inset of Figure 2) The relative dominance of the cation adduct in the matrix-free LDI mass spectra of peptides on the CNTa substrate may be closely related to the ionic environment in which the ions are formed. That is, only a limited amount of hydronium ions, which form in the ionization of the analyte molecules themselves and the solvents, are available on the hydrophobic carbon nanotube surfaces while in the organic acid matrices there exist a large amount of $\mathrm{H}^{+}$ions. The relative intensity ratios of the $\mathrm{Na}^{+}$adduct to the protonated ions also depend upon the nature of the peptides. Probably the ratio reflects the relative values of the $\mathrm{Na}^{+}$ion and proton affinities of the peptide.

In summary, we have demonstrated that relatively small peptides dropped and dried on vertically aligned carbon nanotube arrays can be efficiently desorbed and ionized with $337 \mathrm{~nm}$ laser pulses. Since the vertically aligned CNTa can be easily fabricated in various patterns on substrate by wellestablished semiconductor fabrication technology and can be stored for a long time without decay of performance, it may be an excellent substrate for matrix-free LDI of various small molecules.

Acknowledgments. This work was supported by INHA UNIVERSITY Research Grant.

\section{References}

I. (a) de Hoffmann, E.; Stroobant, V. Mass Spectronetry; Principles and Applications, $2^{\text {nd }}$ ed.; John Wiley \& Sons, Lid.: New York, U.S.A., 2002; p 28. (b) Stump, M. J.; Fleming, R. C.; Gong, W.H.; Jaber, A. J.; Jones, J. J.; Surber, C. W.; Wilkins, C. L. Appl. Spectrosc, Rev. 2002, 37, 275.

2. Tanaka, K.; Waki, H.; Ido, Y.; Akita, S.; Yosida, Y.; Yosida, T. Rapid Commun. Mass Spectrom. 1988, 2, I5I.

3. Wei, J.; Buriak, J. M.; Siuzdak, G Nattre 1999, 399, 243.

4. Shen, Z.; Thomas, J. J.; Averbuj, C.; Broo, K. M.; Engelhard, M.; Crowell, J. E.; Finn, M. G.; Siuzdak, G. Anal. Chem. 2001, 73, 612.

5. (a) Kim, J.; Paek, K.; Kang, W. Bull. Korean Chem. Soc. 2002, 23 , 315, (b) Kim, J.; Kang. W. Bull. Korean Chem. Soc. 2000, 21, $40 \mathrm{l}$.

6. (a) Kim, H.-J.; Lee, J.-K.; Park, S.-J.; Ro, H. W.; Yoo, D. Y,; Yoon, D. Y. Anal. Chem, 2000, 72, 5673. (b) Park, K.-H.; Kim, H.-J. Rapid Commm. Mass Spectrom, 2001, 15, 1494.

7. Xu, S.; Li, Y.; Zou, H.; Qiu, J.; Guo, Z.; Guo, B. Anal. Chen. 2003, 75,6191 .

8. Dai, H. Ace. Chem. Res. 2002, 35, 1035.

9. (a) Fan, S.; Chapline, M. G; Franklin, N. R.; Tombler, T. W; Cassell, A. M.; Dai, H. Science 1999, 283, 512. (b) Sohn, J. I.; Lee, S. Appl. Phys, A 2002, 74, 287.

10. Hagen, A.; Hertel, T. Nano Left. 2003, 3,383. 